\title{
ELASTIC DEFORMATION AND INCLINED MAGNETIC FIELD ON ENTROPY GENERATION FOR WALTER'S LIQUID B FLUID OVER A STRETCHING SHEET
}

\author{
C. Ragavan ${ }^{1}$, S. Munirathinam ${ }^{1}$, M. Govindaraju' ${ }^{2}$, A.K. Abdul Hakeem ${ }^{3}$ \\ B. Ganga \\ ${ }^{1}$ Department of Mathematics, Sri Vidhya Mandir Arts and Science College \\ Uthangarai - 636902, India \\ ${ }^{2}$ Department of Mathematics, Padmavani Arts \& Science College for Women \\ Salem - 636011 , India \\ ${ }^{3}$ Department of Mathematics, Sri Ramakrishna Mission Vidyalaya College of Arts and Science \\ Coimbatore - 641 020, India \\ ${ }^{4}$ Department of Mathematics, Providence College for Women, Coonoor - 643104, India \\ abdulhakeem6@gmail.com
}

Received: 27 September 2018; Accepted: 5 June 2019

\begin{abstract}
An analytical solution is presented for entropy generation on MHD Walter's liquid B fluid over a stretching sheet with elastic deformation. The governing expressions of PDEs are converted into ODEs by suitable transformation which is solved by a hypergeometric function. Plots for velocity, heat transfer, entropy generation and a Bejan number are examined and their behavior is deliberated for several physical parameters. It is noticed that the entropy generation is minimized for an Eckert number and enhanced for an elastic deformation parameter. Moreover, these two parameters on the Bejan number profile have reverse effects.
\end{abstract}

MSC 2010: 28D20, 74F15, 74F10, 76A10

Keywords: entropy, inclined magnetic field, elastic deformation, Walter's liquid B fluid

\section{Nomenclature}

$\begin{array}{ll}a & \text { a constant } \\ \mathrm{Be} & \text { Bejan number } \\ \mathrm{Br} \Omega^{-1} & \text { dimensionless group parameter } \\ C_{f} & \text { skin friction coefficient } \\ E c & \text { Eckert number } \\ \mathrm{Ha} & \text { Hartman number } \\ k_{1} & \text { viscoelastic parameter } \\ k_{0} & \text { elastic parameter } \\ M_{n} & \text { magnetic parameter } \\ N s & \text { entropy generation } \\ N u_{x} & \text { local Nusselt number } \\ \mathrm{Pr} & \text { Prandtl number } \\ \mathrm{Re}_{l} & \text { Reynolds number }\end{array}$


$T \quad$ temperature of the fluid

$T_{\infty} \quad$ free stream temperature

$T_{w} \quad$ temperature at the surface

$u, v$ dimensionless fluid velocity in the $x$ and $y$ direction, respectively

$x, y$ dimensionless cartesian coordinates

Greek letters

$\delta \quad$ elastic deformation parameter

$\eta \quad$ similarity variable

$\mu \quad$ dynamic viscosity of the fluid

$v \quad$ kinemetic viscosity of the fluid

$\rho \quad$ effective density of the fluid

$\sigma \quad$ stream-Boltzmann constant

$\theta$ dimensionless temperature

$k \quad$ thermal condutivity of the fluid

Subscripts

$f \quad$ fluid

$w \quad$ wall constant

\section{Introduction}

The entropy generation system is to compute the destruction of available work and thus diminish the efficiency, consequently the determination of a distribution of entropy production within the fluid flow region can help in raising the system efficiency and achieving the thermal or mechanical design. The entropy production is encountered in many energy-associated applications, such as cooling of modern electronic systems, solar power collectors and geothermal energy systems. The main objective of the system is the minimization of entropy generation. The analytical study of entropy generation on viscoelastic fluid over a stretching sheet embedded in a porous medium was analysed by Baag et al. [1]. They found that the higher value of the Prandtl number minimized the entropy generation. One more analytical solution for entropy generation on viscoelastic fluid flow through a stretching sheet with an inclined magnetic field and non-linear thermal radiation impact was studied by Abdul Hakeem et al. [2]. The impact of entropy generation on viscoelastic fluid through a rotating cylinder was examined by Mirzazadeh et al. [3]. They obtained that the elasticity parameter minimized the entropy generation. The entropy generation on a free convective MHD flow in a parallel-plate vertical channel was investigated by Chin-Chia Liu and Cheng-Ying Lo [4].

The application of an inclined magnetic field in electric conducting viscoelastic fluid has drawn the attention of researchers currently. Animasaun et al. [5] investigated the effect of an induced magnetic field on viscoelastic fluid flow over a stretching sheet. Mair Khan et al. [6] studied the numerical solutions of an inclined mag- 
netic field on the Williamson nanofluid flow past a stretching sheet. Hayat et al. [7] analyzed the heat transfer of nanofluid flow over a stretching sheet with heat generation/absorption and non-linear thermal radiation effects. In controlling momentum and heat transfers in the boundary layer flow of different fluids over a stretching sheet, the applied magnetic field may play an important role [8-15].

The impact of elastic deformation on Walter's liquid B fluid flow past a impermeable stretching sheet was studied by Nandeppanavar et al. [16]. The effect of elastic deformation on a viscoelatic fluid flow past a stretching sheet was analyzed by Khan et al. [17]. Abdul Hakeem et al. [18] reported the effect of elastic deformation on Walter's liquid B fluid flow over a stretching sheet with non-uniform heat source/sink. A numerical study of elastic deformation on nano-second grade fluid flow past a stretching surface was reported by Kalaivanan et al. [19].

Up until now, no study has been made for the analysis of elastic deformation effect for entropy generation on Walter's liquid B fluid flow over a stretching sheet with an inclined magnetic field. So this reality inspires us to propose the same for the current investigation. The main originality of the present work is to analyze the entropy generation of an inclined magnetic field on Walter's liquid B fluid flow over a stretching sheet with elastic deformation. The entropy generation is calculated using the entropy relation by substituting the velocity and temperature fields obtained from the momentum and energy equations.

\section{Formulation of the problem}

Consider a steady, laminar, two-dimensional flow of an incompressible Walter's liquid B fluid past a stretching sheet coinciding with a plane $y=0$ and the flow being confined to $y>0$. The flow is generated due to the stretching of the sheet, caused by the simultaneous application of two equal and opposite forces along the $x$-axis (see Fig. 1). Keeping the origin fixed, the sheet is then stretched with a speed varying linearly with the distance from the slit. The basic boundary layer equations governing the flow of Walter's liquid B fluid can be written as

$$
\begin{aligned}
\frac{\partial u}{\partial x}+\frac{\partial v}{\partial y} & =0 \\
u \frac{\partial u}{\partial x}+v \frac{\partial u}{\partial y} & =v \frac{\partial^{2} u}{\partial y^{2}}-k_{0}\left\{u \frac{\partial^{3} u}{\partial x \partial y^{2}}+v \frac{\partial^{3} u}{\partial y^{3}}+\frac{\partial u}{\partial x} \frac{\partial^{2} u}{\partial y^{2}}-\frac{\partial u}{\partial y} \frac{\partial^{2} u}{\partial x \partial y}\right\} \\
& -\frac{\sigma B_{0}^{2}}{\rho} u \sin ^{2} \gamma \\
u \frac{\partial T}{\partial x}+v \frac{\partial T}{\partial y} & =\frac{k}{\rho c_{p}} \frac{\partial^{2} T}{\partial y^{2}}-\frac{\delta k_{0}}{c_{p}}\left\{\frac{\partial u}{\partial y} \frac{\partial}{\partial y}\left(u \frac{\partial u}{\partial x}+v \frac{\partial u}{\partial y}\right)\right\}
\end{aligned}
$$

where $(u, v)$ are velocity components along $x$ and $y$ axes respectively. 


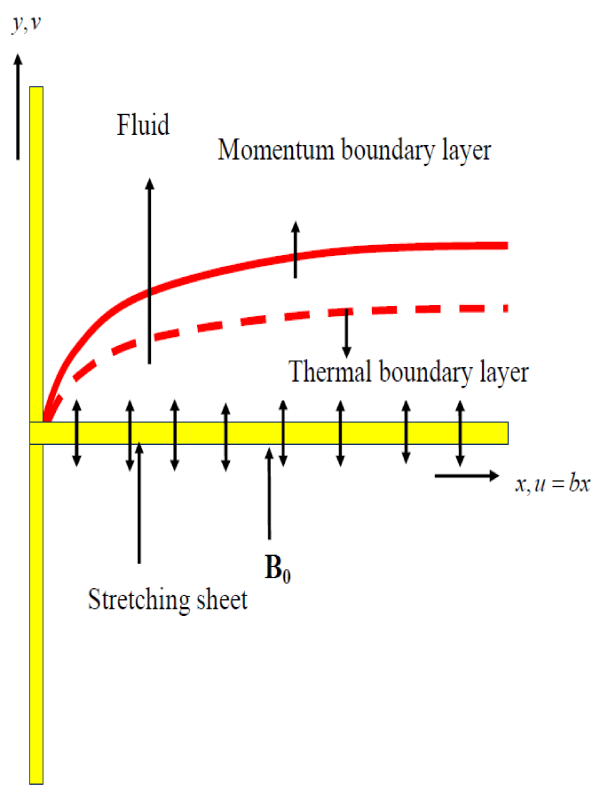

Fig. 1. Schematic diagram of flow and physical model

The assumed boundary conditions are

$$
\begin{array}{cc}
u=b x, v=0 & T=T_{w}=T_{\infty}+A\left(\frac{x}{l}\right)^{2} \text { at } y=0 \\
u \rightarrow \infty, & T \rightarrow T_{\infty} \quad \text { as } y \rightarrow \infty
\end{array}
$$

With $b>0$, this is stretching rate, $T_{w}$ and $T_{\infty}$ represent the temperature of the wall and temperature outside the dynamic region respectively. The constant $A$ depends on the thermal properties of the liquid and $l=\sqrt{\frac{v}{b}}$ is a characteristic length.

We define the following new variables

$$
u=b x f_{\eta}(\eta) v=-(b v)^{\frac{1}{2}} f(\eta), \quad \eta=\left(\frac{b}{v}\right)^{\frac{1}{2}} y \quad \theta(\eta)=\frac{T-T_{\infty}}{T_{w}-T_{\infty}}
$$

Making use of Eq. (5), continuity Eq. (1) is identically satisfied while Eqs. (2) and (3) take the following form:

$$
\begin{array}{r}
f_{\eta}^{2}-f f_{\eta \eta}=f_{\eta \eta \eta}-k_{1}\left[2 f_{\eta} f_{\eta \eta \eta}-f f_{\eta \eta \eta \eta}-f_{\eta \eta}^{2}\right]-M_{n} f_{\eta} \sin ^{2} \gamma \\
\theta_{\eta \eta}+\operatorname{Pr} f \theta_{\eta}-2 \operatorname{Pr} f_{\eta} \theta=-E c \operatorname{Pr}\left[f_{\eta \eta}^{2}-\delta k_{1} f_{\eta \eta}\left(f_{\eta} f_{\eta \eta}-f f_{\eta \eta \eta}\right)\right]
\end{array}
$$

where $k_{1}=\frac{k_{0} b}{v}, M_{n}=\frac{\sigma B_{0}^{2}}{\rho b}, E c=\frac{b^{2} l^{2}}{A c_{p}}$ and $\operatorname{Pr}=\frac{\mu c_{p}}{k}$. 
Similarly boundary conditions become,

$$
\begin{array}{r}
f_{\eta}(\eta)=1, \quad f(\eta)=0, \quad \theta(\eta)=1 \text { at } \eta=0 \\
f_{\eta}(\eta) \rightarrow 0, \quad \theta(\eta) \rightarrow \infty \text { as } \eta \rightarrow \infty
\end{array}
$$

Here boundary conditions are one less in number required to solve the flow problem uniquely. Following Abdul Hakeem et al. [18], the solution of Eq. (6) with boundary conditions (8) can be written in the form:

$$
f(\eta)=\frac{1-e^{-\alpha \eta}}{\alpha}, \text { with } \alpha=\sqrt{\frac{1+M_{n} \sin ^{2} \gamma}{1-k_{1}}}
$$

Obviously, $0<k_{1}<1$.

Using the solution (9) in Eq. (5), the velocity components obtained in the form

$$
u=b x e^{-\alpha \eta}, \text { and } v=-\sqrt{b v}\left\{\frac{1-e^{-\alpha \eta}}{\alpha}\right\} .
$$

The wall shearing stress on the surface of the stretching sheet is given by

$$
\tau_{w}=\left[v \frac{\partial u}{\partial y}-k_{0}\left(u \frac{\partial^{2} u}{\partial x \partial y}-2 \frac{\partial u}{\partial x} \frac{\partial u}{\partial y}\right)\right]_{y=0}
$$

The local skin-friction coefficient or the frictional drag is given by

$$
C_{f}=\frac{\tau_{w}}{\frac{1}{2} \rho u_{w}^{2}}=2 \operatorname{Re}_{x}^{-1 / 2}\left(1-3 k_{1}\right) f_{\eta \eta}(0) .
$$

\subsection{Solution of thermal transport}

The solution of Eq. (7), subject to boundary conditions (8), can be obtained in terms of hypergeometric function [18] as

$$
\begin{array}{r}
\theta(\eta)=C_{2}\left(e^{-\alpha \eta}\right)^{\frac{a_{0}+b_{0}}{2}} M\left(\frac{a_{0}+b_{0}-4}{2}, 1+b_{0},\right. \\
\left.\frac{-P r}{\alpha^{2}} e^{-\alpha \eta}\right) \\
+C_{1} e^{-2 \alpha \eta}
\end{array}
$$

Where $a_{0}=b_{0}=\frac{P r}{\alpha^{2}}, C_{1}=\frac{-E c P r\left(1-\delta k_{1}\right)}{2\left(2-a_{0}\right)}, C_{2}=\frac{1-C_{1}}{M\left(\frac{a_{0}+b_{0}-4}{2}, 1+b_{0}, \frac{-P r}{\alpha^{2}}\right)}$

The non-dimensional wall temperature gradient derived from Eq. (13) reads as

$$
\begin{array}{r}
\theta^{\prime}(0)=C_{2}\left[-\alpha\left(\frac{a_{0}+b_{0}}{2}\right) M\left(\frac{a_{0}+b_{0}-4}{2}, 1+b_{0}, \frac{-P r}{\alpha^{2}}\right)\right. \\
\left.+\frac{\operatorname{Pr}}{\alpha}\left(\frac{a_{0}+b_{0}-4}{2\left(1+b_{0}\right)}\right) M\left(\frac{a_{0}+b_{0}-2}{2}, 2+b_{0}, \frac{-P r}{\alpha^{2}}\right)\right]-2 \alpha C_{1}
\end{array}
$$




\section{Entropy generation analysis}

According to Woods [20], the local volumetric rate of entropy generation in the presence of a magnetic field is given by

$$
S_{G}=\frac{k}{T_{\infty}^{2}}\left[\left(\frac{\partial T}{\partial x}\right)^{2}+\left(\frac{\partial T}{\partial y}\right)^{2}\right]+\frac{\mu}{T_{\infty}}\left(\frac{\partial u}{\partial y}\right)+\frac{\sigma B_{0}^{2}}{T_{\infty}} u^{2} \sin ^{2} \gamma
$$

Equation (15) puts on display the three entropy generation sources. The heat transport covering a finite temperature difference that generates entropy is given with the first term of Eq. (15), viscous dissipation creating local entropy generation is the second term, likewise, the third term represents the local entropy generated by means of the magnetic field effect. It is appropriate to define dimensionless number for entropy generation rate $N_{S}$. This number is defined by dividing the local volumetric entropy generation rate $S_{G}$ to a characteristic entropy generation rate $S_{G_{0}}$. For the prescribed boundary condition, the characteristic entropy generation rate is

$$
S_{G_{0}}=\frac{k(\Delta T)^{2}}{l^{2} T_{\infty}^{2}}
$$

therefore, the entropy genetration number is

$$
N_{s}=\frac{S_{G}}{S_{G_{0}}}
$$

Using Eqs. (13), (15) and (17), the entropy generation number is given by

$$
N_{s}=\frac{4}{X^{2}} \theta^{2}(\eta)+R e_{l} \theta^{\prime 2}(\eta)+R e_{l} \frac{B r}{\Omega} f^{\prime \prime 2}(\eta)+\frac{B r H a^{2}}{\Omega} f^{\prime 2}(\eta) \sin ^{2} \gamma
$$

where $R e_{l}$ and $\mathrm{Br}$ are respectively the Reynolds number and the Brinkman number. $\Omega$ and $H a$ are respectively the dimensionless temperature difference and the Hartman number. These numbers are given by the following relationships

$$
R e_{l}=\frac{u_{l} l}{v}, \quad B r=\frac{\mu u_{p}^{2}}{k \Delta T} \quad \Omega=\frac{\Delta T}{T_{\infty}}, \quad H a=B_{0} l \sqrt{\frac{\sigma}{\mu}}
$$

\section{Bejan number analysis}

In the flow region, the entropy generation number $(N s)$ represents the entropy generation distribution. Bejan number $(B e)$ relating the thermal irreversibility importance to total irreversibility is very much needed in the energy optimization problem 
and is defined as [21]

$$
B e=\frac{\frac{k}{T_{\infty}^{2}}\left(\frac{\partial T}{\partial x}\right)^{2}}{\frac{k}{T_{\infty}^{2}}\left(\frac{\partial T}{\partial y}\right)^{2}+\frac{\mu}{T_{\infty}}\left(\frac{\partial u}{\partial y}\right)+\frac{\sigma B_{0}^{2}}{T_{\infty}} u^{2} \sin ^{2} \gamma}
$$

Using Eq. (5), Eq. (23) reduces to the following form

$$
B e=\frac{\frac{4}{X^{2}} \theta^{2}(\eta)}{\frac{4}{X^{2}} \theta^{2}(\eta)+\operatorname{Re}_{l} \theta^{\prime 2}(\eta)+\operatorname{Re}_{l} \frac{B r}{\Omega} f^{\prime \prime 2}(\eta)+\frac{B r H a^{2}}{\Omega} f^{\prime 2}(\eta) \sin ^{2} \gamma}
$$

\section{Result and discussion}

The physical revelation of different non-dimensionless parameters on the velocity, temperature, entropy generation and Bejan number are illustrated in this section. In order to verify the accuracy of the present results, we have ccompared the values of the reduced Nusselt number with Turkyilmazoglu [22] and Datti et al. [23]. The comparisons are found to be good, which is shown in Table 1. Variation of a magnetic parameter, inclined angle, and viscoelastic parameter on $f(\eta)$ is presented in Figure 2. From this figure the presence of these parameters diminishes the movement of the fluid. Figure 3 describes the impact of the magnetic parameter, viscoelastic parameter, elastic deformation and Prandtl number on $\theta(\eta)$. It is revealed that as the increasing values of magnetic parameter, viscoelastic parameter, and Prandtl number increments $\theta(\eta)$ in contrast to elastic deformation which brings down $\theta(\eta)$. The existence of the magnetic parameter, viscoelastic parameter, and Prandtl number physically enhances the temperature. This enhancement is because of more heat that is produced during the random motion of the fluid particles. In this way the temperature of the fluid further improves. But elastic deformation reduces the temperature distribution.

Table 1. Comparison of results for the reduced Nusselt number $-\theta^{\prime}(0)$. When $\gamma=90, E c=\delta=0$

\begin{tabular}{cccccc}
\hline$M_{n}$ & $\operatorname{Pr}$ & $k_{1}$ & Turkyilmazoglu [22] & Datti et al. [23] & Present value \\
\hline 1 & 0 & 0 & 1.333333 & 1.3333 & 1.333333 \\
1 & 1 & 0 & 1.215773 & 1.2158 & 1.215770 \\
1 & 1 & 0.2 & - & 1.1687 & 1.168700 \\
0 & 5 & 0 & 3.31648 & - & 3.316480 \\
0 & 10 & 0 & 4.69021 & - & 4.690210 \\
\hline
\end{tabular}

The variations of magnetic parameter and the inclined angle on Ns are shown in Figures 4 and 5 respectively. From these figures, both magnetic parameter and 


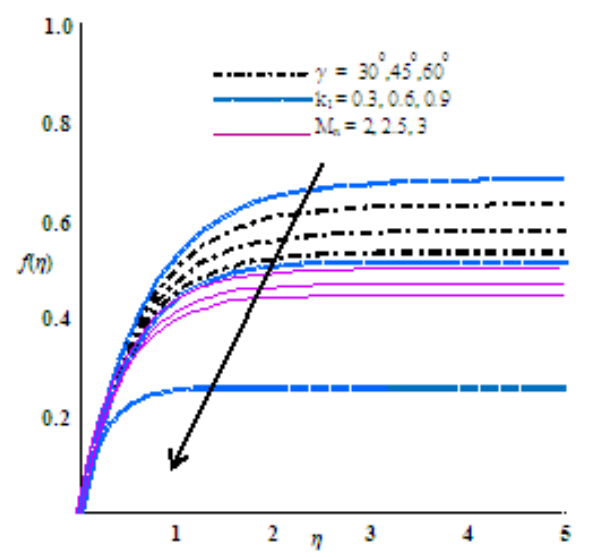

Fig. 2. Effect of aligned angle, viscoelasticity and magnetic parameters on velocity profile with $\gamma=45^{\circ}, k_{1}=0.4, M_{n}=1.0$

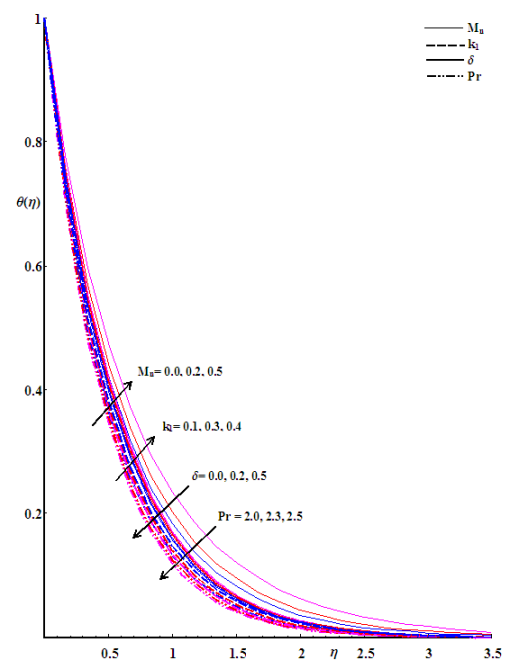

Fig. 3. Effect of magnetic parameter, viscoelastic parameter, elastic deformation, Prandtl number on temperature distribution with $k_{1}=0.1, M_{n}=1.0, \gamma=45^{\circ}, E c=0.4, \operatorname{Pr}=3.0, \delta=1.0$

inclined angle upgrades entropy generation. This means that the enhancing value of the angle parameter strengthens the magnetic field, so magnetic force opposes the fluid motion at the same time enhances the temperature of the fluid. Thus the entropy generation rate increases with rising value of magnetic parameter.

Figure 6 relates entropy generation to viscoelastic parameter, it is observed from this figure that increasing values of viscoelastic parameter slightly amplifies the entropy generation. Physically, viscoelastic fluid increases the entropy production. In Figure 7 it is examined that the entropy generation rate improves through higher values of elastic deformation while at the same time the opposite effect is observed far away from the sheet. The effect of the Eckert number on entropy generation is shown 


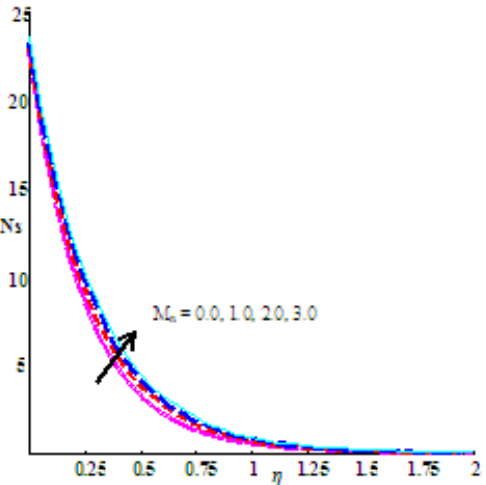

Fig. 4. Effect of magnetic parameter on entropy generation with $k_{1}=0.4, \operatorname{Pr}=3.0, \gamma=45^{\circ}$, $E c=0.4, \delta=1.0, \mathrm{Ha}=1.0, \mathrm{Br} \Omega^{-1}=1.0$

$$
R e_{l}=1.0, X=0.5
$$

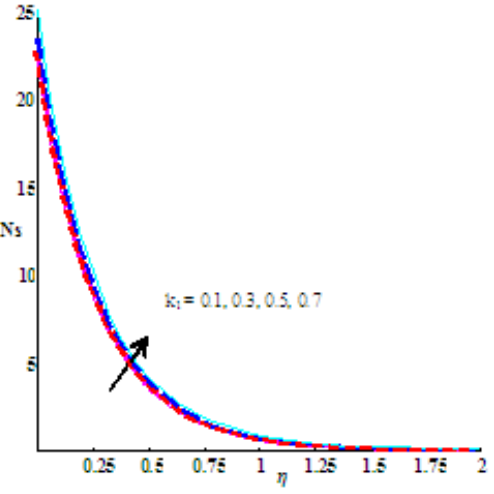

Fig. 6. Effect of viscoelastic parameter on entropy generation with $M_{n}=1.0, \operatorname{Pr}=3.0, \gamma=45^{\circ}$, $E c=0.4, \delta=1.0, \mathrm{Ha}=1.0, \mathrm{Br} \Omega^{-1}=1.0$, $R e_{l}=1.0, X=0.5$

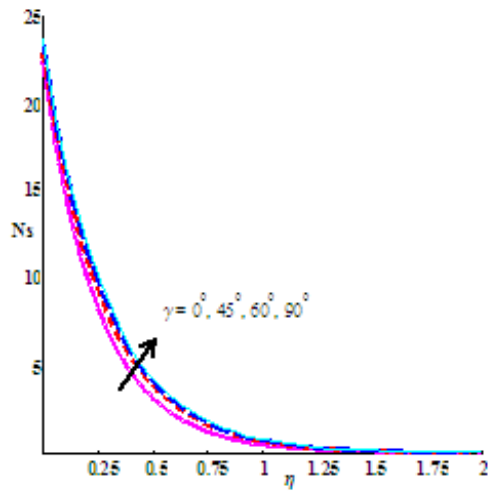

Fig. 5. Effect of angle parameter on entropy generation with $k_{1}=0.4, \operatorname{Pr}=3.0, M_{n}=1$, $E c=0.4, \delta=1.0, H a=1.0, B r \Omega^{-1}=1.0$, $R e_{l}=1.0, X=0.5$

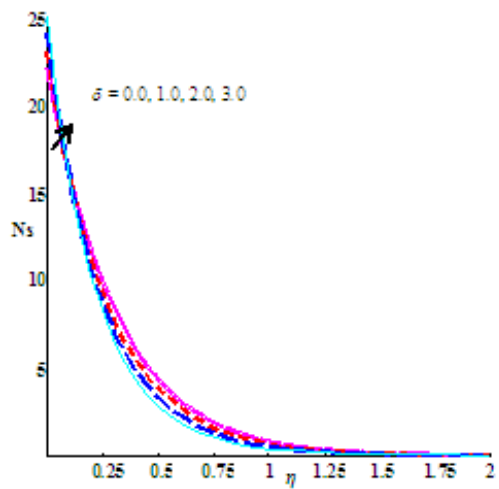

Fig. 7. Effect of elastic deformation parameter on entropy generation with $k_{1}=0.4, \operatorname{Pr}=3.0$, $\gamma=45^{\circ}, E c=0.4, M_{n}=1.0, H a=1.0$, $B r \Omega^{-1}=1.0, \operatorname{Re}_{l}=1.0, X=0.5$

in Figure 8. It is ultimately seen that higher values of the Eckert number minimized the entropy production.

The distribution of the Bejan number with respect to the magnetic parameter is shown in Figure 9. It is clear that the magnetic parameter increases the Bejan number in this manner the irreversibility due to heat transfer is enhanced. The influences of the viscoelastic parameter and Eckert number on the Bejan number is represented by Figures 10 and 11 respectively. These figures reveal that the Bejan number enhances both the viscoelastic parameter and Eckert number, relating to the fact that heat is a form of disorganized energy. Figure 12 represents the effect of elastic deformation on the Bejan number. It is clear that the increasing values of the elastic parameter decreases the Bejan number. 


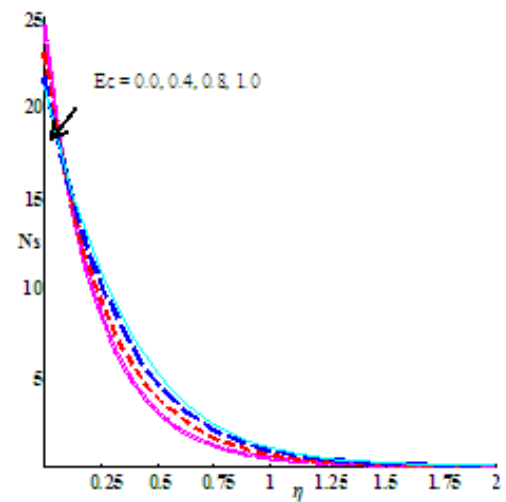

Fig. 8. Effect of Eckert number on entropy generation with $k_{1}=0.4, \operatorname{Pr}=3.0, \gamma=45^{\circ}$ $M_{n}=1.0, \delta=1.0, H a=1.0, B r \Omega^{-1}=1.0$, $\operatorname{Re}_{l}=1.0, X=0.5$

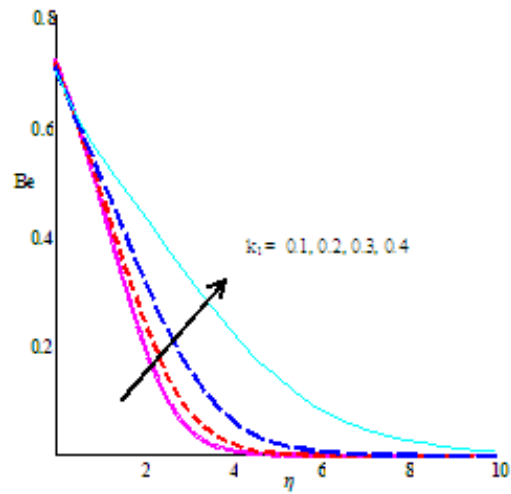

Fig. 10. Effect of viscoelastic parameter on the Bejan number with $M_{n}=1.0, \operatorname{Pr}=3.0, \gamma=45^{\circ}$, $E c=0.4, \delta=1.0, H a=1.0, B r \Omega^{-1}=1.0$, $R e_{l}=1.0, X=0.5$

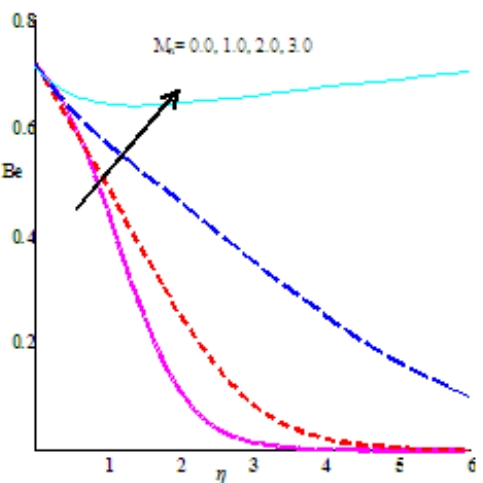

Fig. 9. Effect of magnetic parameter on the Bejan number with $k_{1}=0.4, \operatorname{Pr}=3.0, \gamma=45^{\circ}$, $E c=0.4, \delta=1.0, \mathrm{Ha}=1.0, \mathrm{Br} \Omega^{-1}=1.0$, $\operatorname{Re}_{l}=1.0, X=0.5$

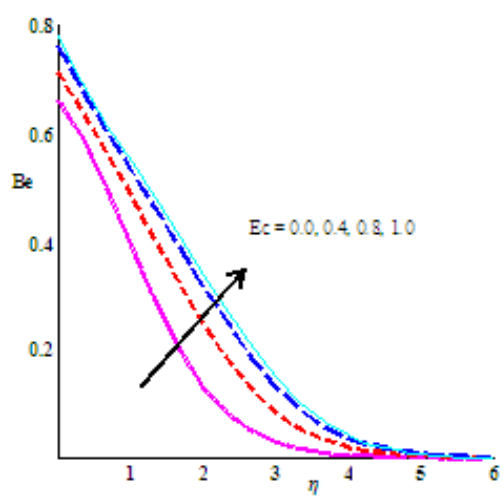

Fig. 11. Effect of Eckert number on the Bejan number with $k_{1}=0.4, \operatorname{Pr}=3.0, \gamma=45^{\circ}$, $M_{n}=1.0, \delta=1.0, \mathrm{Ha}=1.0, \mathrm{Br} \Omega^{-1}=1.0$, $\operatorname{Re}_{l}=1.0, X=0.5$

Figure 13 represents the effect of the viscoelastic parameter on the skin friction coefficient. It is clear that the skin friction coefficient increases as the viscoelastic parameter increases. The effect of the elastic deformation parameter, viscoelastic parameter and Prandtl number on the reduced Nusselt number are plotted in Figure 14. It is noteworthy that the reduced Nusselt number decreases with the increasing values of the elastic deformation parameter, viscoelastic parameter and Prandtl number.

The values of $f_{\eta \eta}(0)$ and $\theta_{\eta}(0)$ are tabulated in Tables 2 and 3. From these Tables, it clear that the skin friction increases with $k_{1}, M_{n}$ and $\gamma$. The reduced Nusselt number increases with $\operatorname{Pr}$ and $\delta$ and decreases with $k_{1}, M_{n}, \gamma$ and $E c$. 


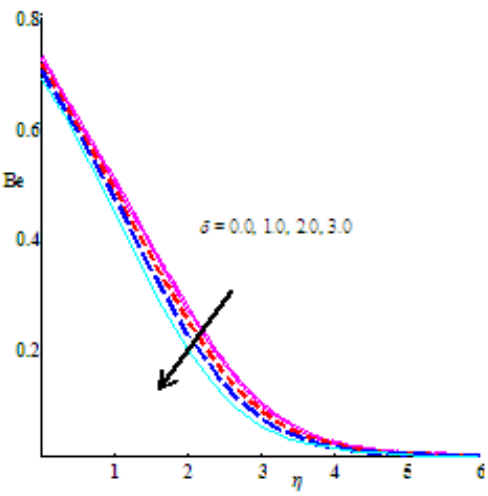

Fig. 12. Effect of elastic deformation on the Bejan number with $k_{1}=0.4, \operatorname{Pr}=3.0, \gamma=45^{\circ}$, $E c=0.4, M_{n}=1.0, \mathrm{Ha}=1.0, \mathrm{Br} \Omega^{-1}=1.0$,

$$
R e_{l}=1.0, X=0.5
$$

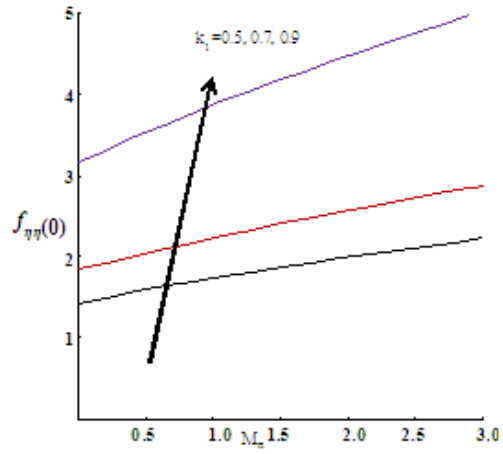

Fig. 13. Local skin friction coefficient for various values of $k_{1}$

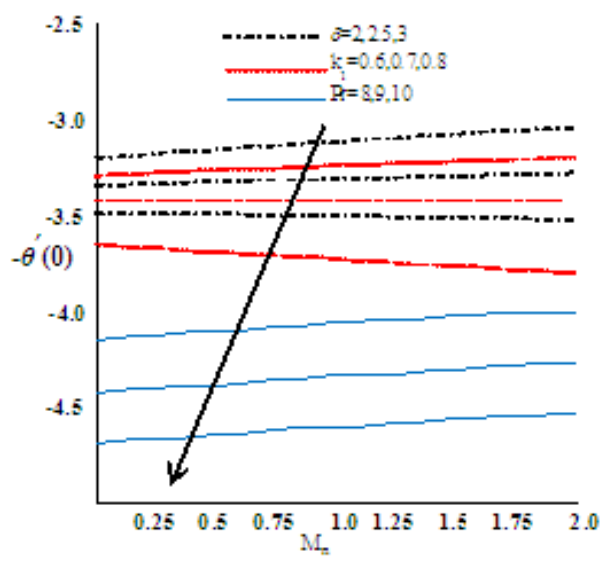

Fig. 14. Effect of the elastic deformation parameter, viscoelastic parameter and Prandtl number on the Nusselt number with $\gamma=45^{\circ}, \delta=2, E c=0.3, k_{1}=0.5, \operatorname{Pr}=5$

\section{Conclusions}

Here we investigate the effect of elastic deformation for entropy generation on Walter's liquid B fluid flow over a stretching sheet with an inclined magnetic field and also compute the entropy generation with the Bejan number. The various physical parameters on velocity, temperature, entropy generation and Bejan number are analyzed. The most important results are indicated as:

- The velocity profile is a decreasing function of the magnetic parameter, viscoelastic parameter and inclined angle.

- The temperature profile enhances with the magnetic parameter, viscoelastic parameter and Prandtl number and it decreases with elastic deformation. 
Table 2. Values of $f_{\eta \eta}(0)$ for several of the parameters

\begin{tabular}{cccc}
\hline$k_{1}$ & $M_{n}$ & $\gamma$ & $f_{\eta \eta}(0)$ \\
\hline 0.0 & 1.0 & $90^{\circ}$ & 1.41421 \\
0.2 & & & 1.58114 \\
0.4 & & & 1.82574 \\
0.2 & 0.0 & & 1.11803 \\
& 1.0 & & 1.58114 \\
& 2.0 & & 1.93649 \\
& & $0^{\circ}$ & 1.11803 \\
& & $45^{\circ}$ & 1.36931 \\
& & $90^{\circ}$ & 1.58114 \\
\hline & & &
\end{tabular}

Table 3. Values of $\theta_{\eta}(0)$ for several of the parameters

\begin{tabular}{ccccccc}
\hline $\operatorname{Pr}$ & $k_{1}$ & $M_{n}$ & $\gamma$ & $\mathrm{Ec}$ & $\delta$ & $\theta_{\eta}(0)$ \\
\hline 1.0 & 0.2 & 1.0 & $90^{\circ}$ & 0.4 & 1.0 & 0.96934 \\
2.0 & & & & & & 1.48548 \\
3.0 & & & & & & 1.86521 \\
& 0.0 & & & & & 1.88104 \\
& 0.2 & & & & & 1.86521 \\
& 0.4 & & & & & 1.83515 \\
& 0.2 & 0.0 & & & & 2.18763 \\
& & 1.0 & & & & 1.86521 \\
& & 2.0 & & & & 1.60316 \\
& & 1.0 & $0^{\circ}$ & & & 2.18763 \\
& & & $45^{\circ}$ & & & 1.90844 \\
& & & $90^{\circ}$ & & & 1.89581 \\
& & & & 0.0 & & 2.35161 \\
& & & & 0.2 & & 2.10841 \\
& & & & 0.4 & & 1.86521 \\
& & & & & 0.0 & 1.74361 \\
& & & & & 2.0 & 1.86521 \\
& & & & & &
\end{tabular}

- Entropy generation enhances with the magnetic parameter, inclined angle, viscoelastic parameter and elastic deformation whereas it decreases with the Eckert number.

- The Bejan number increases with the magnetic parameter, viscoelastic parameter and the Eckert number and it decreases with elastic deformation. 


\section{Acknowledgment}

One of the authors (A.K. Abdul Hakeem) gratefully acknowledges the financial support of minor research project, UGC, New Delhi, India under UGC/SERO/MRP6729/16 for pursuing this work.

\section{References}

[1] Baag, S., Mishra, S.R., Dash, G.C., \& Acharya, M.R. (2017). Entropy generation analysis for viscoelastic MHD flow over a stretching sheet embedded in a porous medium. Ain Shams Engineering Journal, 8, 623-632.

[2] Abdul Hakeem, A.K., Govindaraju, M., \& Ganga, B. (2018). Influence of inclined Lorentz forces on entropy generation analysis for viscoelastic fluid over a stretching sheet with nonlinear thermal radiation and heat source/sink. Journal of Heat and Mass Transfer Research, DOI: 10.22075/JHMTR.2018.13611.1198.

[3] Mirzazadeh, M., Shafaei, A., \& Rashidi, F. (2008). Entropy analysis for non-linear viscoelastic fluid in concentric rotating cylinders. International Journal of Thermal Sciences, 47, 1701-1711.

[4] Chin-Chia Liu, \& Cheng-Ying Lo,(2012). Numerical analysis of entropy generation in mixedconvection MHD flow in vertical channel. International Communications in Heat and Mass Transfer, 39, 1354-1359.

[5] Animasaun, I.L., Raju, C.S.K., \& Sandeep, N. (2016). Unequal diffusivities case of homogeneous heterogeneous reactions within viscoelastic fluid flow in the presence of induced magnetic-field and nonlinear thermal radiation. Alexandria Engineering Journal, 55, 1595-1606.

[6] Mair Khan, Malik, M.Y., Salahuddin, T., \& Arif. Hussian, (2018). Heat and mass transfer of Williamson nanofluid flow yield by an inclined Lorentz force over a nonlinear stretching sheet. Results in Physics, 8, 862-868.

[7] Tasawar Hayat, Sajid Qayyum, Ahmed Alsaedi,\& Anum Shafiq. (2016). Inclined magnetic field and heat source/sink aspects in flow of nanofluid with nonlinear thermal radiation. International Journal of Heat and Mass Transfer, 103, 99-107.

[8] Farooq, M., Ijaz Khan, M., Waqas, M., Hayat, T., Alsaedi, A., \& M. Imran Khan. (2016). MHD stagnation point flow of viscoelastic nanofluid with non-linear radiation effects. Journal of Molecular Liquids, 221, 1097-1103.

[9] Sheikholeslami, M., Hari R. Kataria, \& Akhil S. Mittal. (2018). Effect of thermal diffusion and heat-generation on MHD nanofluid flow past an oscillating vertical plate through porous medium. Journal of Molecular Liquids, 257, 12-25.

[10] Sheikholeslami, M., \& Rokni, H.B. (2017). Influence of melting surface on MHD nanofluid flow by means of two phase model. Chinese Journal of Physics, 55(4), 1352-1360.

[11] Abderrahim Wakif., Zoubair Boulahia., Farhad Ali., Mohamed R. Eid, \& Rachid Sehaqui. (2018). Numerical analysis of the unsteady natural convection MHD Couette nanofluid flow in the presence of thermal radiation using single and two-phase nanofluid models. International Journal of Applied and Computational Mathematics, 4(81), 1-27.

[12] Mohamed R. Eid., \& Kasseb L. Mahny. (2017). Unsteady MHD heat and mass transfer of a non-Newtonian nanofluid flow of a two-phase model over a permeable stretching wall with heat generation/absorption. Advanced Powder Technology, 28(11), 3063-3073.

[13] Mohamed R. Eid. (2016). Chemical reaction effect on MHD boundary-layer flow of two-phase nanofluid model over an exponentially stretching sheet with a heat generation. Journal of Molecular Liquids, 220, 718-725. 
[14] Mohamed R. Eid., \& Makinde, O.D. (2018). Solar radiation effect on a magneto nanofluid flow in a porous medium with chemically reactive species. International Journal of Chemical Reactor Engineering, 20170212.

[15] Mohamed R. Eid, Kasseb L. Mahny, Muhammad, T., \& Sheikholeslami, M. (2018). Numerical treatment for Carreau nanofluid flow over a porous nonlinear stretching surface. Results in Physics, 8, 1185-1193.

[16] Nandeppanavar, M.M., Subhas Abel, M., \& Jagadish Tawade. (2010). Heat transfer in a Walter's liquid B fluid over an impermeable stretching sheet with non-uniform heat source/sink and elastic deformation. Commun. Nonlinear Sci. Numer. Simulat, 15, 1791-1802.

[17] Sujit Kumar Khan, Subhas Abel, M., \& Ravi M. Sonth. (2003). Visco-elastic MHD flow, heat and mass transfer over a porous stretching sheet with dissipation of energy and stress work. Heat and Mass Transfer, 40, 47-57.

[18] Abdul Hakeem, A.K., Vishnu Ganesh, N., \& Ganga, B. (2014). Effect of heat radiation in a Walter's liquid B fluid over a stretching sheet with non-uniform heat source/sink and elastic deformation. Journal of King Saud University - Engineering Sciences, 26(2), 168-175.

[19] Kalaivanan, R., Ganga, B., Vishnu Ganesh, N., \& Abdul Hakeem, A.K. (2018). Effect of elastic deformation on nano-second grade fluid flow over a stretching surface. Frontiers in Heat and Mass Transfer, 10, 20-29.

[20] Woods, L.C. (1975). Thermodynamics of fluid Systems. Oxford: Oxford University Press.

[21] Afridi, M.I., Qasim, M., Ilyas Khan, \& Tlili, I. (2018). Entropy generation in MHD mixed convection stagnation-point flow in the presence of Joule and frictional heating. Case Studies in Thermal Engineering, 12, 292-300.

[22] Turkyilmazoglu, M. (2011). Analytic heat and mass transfer of the mixed hydrodynamic/thermal slip MHD viscous flow over a stretching sheet. International Journal of Mechanical Sciences, 53, 886-896.

[23] Datti, P.S., Prasad, K.V., Subhas Abel, M., \& Ambuja Joshi. (2004). MHD visco-elastic fluid flow over a non-isothermal stretching sheet. International Journal of Engineering Science, 42, 935-946. 